\title{
EFEITOS CARDIORRESPIRATÓRIOS FRENTE À POSIÇÃO DO CORPO EM RECÉM-NASCIDOS PRÉ-TERMO SUBMETIDOS AO AUMENTO DO VOLUME GÁSTRICO
}

\author{
Gisley de PÁDUA ${ }^{1}$, Edson Zangiacomi MARTINEZ ${ }^{1}$ e Marisa Afonso de Andrade BRUNHEROTTI²
}

RESUMO - Contexto - O aumento do volume gástrico observado na alimentação por gavagem traz consequências ao recém-nascido pré-termo, alterando indicadores da função respiratória. Objetivo - Investigar as alterações no sistema cardiorrespiratório nas diferentes posições do corpo de recém-nascidos prematuros submetidos ao aumento do volume gástrico através da alimentação por gavagem. Métodos - Trata-se de um ensaio aleatório, tipo "crossover". Foram estudados 16 recém-nascidos de 31 a 34 semanas de idade gestacional e peso menor ou igual a 2.500 g. Foram incluídos os recém-nascidos com 7 a 10 dias de vida, alimentados via sonda orogástrica (fórmula artificial padronizada e/ou leite materno ordenhado no banco de leite do Hospital Santa Casa de Misericórdia de Franca, SP) de 3 em 3 horas, volume total de $150 \mathrm{~mL} / \mathrm{kg} /$ dia, sem oxigenoterapia suplementar. Foi utilizado a cada gavagem um posicionamento diferente e elevado a $30^{\circ}$, tal como: lateral direito, lateral esquerdo, pronação e supinação. Foram observadas as variáveis: frequência respiratória e cardíaca, saturação de oxigênio, tiragem de intercostais, batimento de asa nasal e gemência. Estas variáveis foram coletadas de 2 em 2 minutos, a partir de 5 minutos, antecedendo a gavagem e até 5 minutos após. Resultados - O grupo estudado apresentou média de 32 semanas de idade gestacional (desvio-padrão 1,3), peso médio de $1.722 \mathrm{~g}$ (desvio-padrão 276,3). Em supinação e lateral esquerdo, a frequência respiratória média apresentou-se com valores mais altos e a saturação de oxigênio média apresentou os menores valores. Em lateral direito e pronação os níveis de frequência cardíaca média foram mais baixos e estáveis. Os posicionamentos lateral direito e pronação apresentaram maior ausência de tiragem intercostal, batimento de asa nasal e gemência. Conclusão - Os decúbitos lateral direito e pronação demonstraram maior repercussão positiva (menor aumento da frequência cardíaca, da frequência respiratória, menor diminuição da saturação de oxigênio, menos presença de tiragem de intercostais, batimento de asa nasal e gemência) nos efeitos cardiorrespiratórios, sendo as posições lateral esquerda e supinação aquelas de maiores efeitos negativos nos recém-nascidos submetidos ao aumento do volume gástrico.

DESCRITORES - Prematuro. Postura. Sistema cardiovascular. Respiração. Nutrição enteral.

\section{INTRODUÇÃO}

As alterações no sistema cardiorrespiratório nos recém-nascidos $(\mathrm{RN})$ durante o aumento do volume gástrico, como na alimentação por gavagem, incluem: aumento da frequência respiratória, aumento da frequência cardíaca, fadiga muscular, apnéias, cianose, hipoxemia e hipercapnia ${ }^{(4,7,12,19)}$.

Posicionamento corporal adequado apresenta grande influência sobre a biomecânica da caixa torácica, pois serve como ponto de apoio à musculatura respiratória, ocasiona maior ponto de apoio e contratilidade muscular, altera a pressão pleural, aumenta o volume de ar pulmonar, gerando menor esforço respiratório e menor desvantagem mecânica ao sistema respiratório ${ }^{(16,}$ ${ }^{23)}$. O posicionamento direcionado aos $\mathrm{RN}$ prematuros promove uma reorganização sensorial, sono tranquilo, menor gasto energético e menor alteração no equilíbrio muscular postural, promovendo menor índice de complicações futuras no desenvolvimento ${ }^{(16,23,30)}$.

O conhecimento do posicionamento de decúbito ideal no momento da alimentação por via enteral é de grande importância para promover menores repercussões negativas ao sistema cardiorrespiratório dos $\mathrm{RN}$.

Desta maneira, o objetivo deste estudo foi analisar a resposta aos posicionamentos através dos efeitos cardiorrespiratórios em RN pré-termo submetidos ao aumento do volume gástrico durante a gavagem.

\section{MÉTODOS}

O presente estudo é um ensaio clínico aleatório com delineamento prospectivo, do tipo "crossover", conduzido nas dependências da Unidade Neonatal,

Trabalho foi realizado no Departamento de Medicina Social. Faculdade de Medicina de Ribeirão Preto da Universidade de São Paulo. 'Departamento de Medicina Social, Faculdade de Medicina de Ribeirão Preto, SP; ${ }^{2}$ Universidade de Franca, SP.

Correspondência: Prof. Edson Zangiacomi Martinez - Departamento de Medicina Social - Faculdade de Medicina de Ribeirão Preto - Universidade de São Paulo - Av. Bandeirantes, 3900 - 14049-900 - Ribeirão Preto, SP. E-mail: edson@fmrp.usp.br 
Berçário Externo, Centro de Terapia Intensiva Infantil e Mãe Canguru do Hospital Santa Casa de Misericórdia de Franca, SP, através do Departamento de Medicina Social da Faculdade de Medicina de Ribeirão Preto da Universidade de São Paulo, (FMRP-USP) Ribeirão Preto, SP, de abril a novembro de 2006.

A pesquisa obteve a aprovação do Comitê de Ética em Pesquisa da FMRP-USP (Processo no 15588 2005) e pelo Conselho Técnico do Hospital Santa Casa de Misericórdia de Franca. Os termos de consentimento livre e esclarecido foram apresentados, aceitos e assinados pelos pais e/ou responsáveis pelos menores.

Numa fase preliminar, foi realizado um teste piloto em dois $\mathrm{RN}$, com o objetivo de verificar as rotinas de decúbitos aos quais seriam submetidos, possibilitando assim, os reajustes ambientais e a homogeneidade da coleta. Com base nos resultados obtidos e na teoria estatística de modelos de efeitos mistos ${ }^{(18)}$, estimou-se que $16 \mathrm{RN}$ seriam suficientes para atender o objetivo do presente estudo, considerando um poder de $80 \%$ e nível de significância de $5 \%$.

Foram incluídos apenas os $\mathrm{RN}$ pré-termos moderados de 31 a 34 semanas, conforme o método CAPURRO et al. ${ }^{(5)}$, que utilizavam alimentação via sonda orogástrica (fórmula artificial padronizada e/ou leite materno ordenhado no banco de leite do Hospital Santa Casa de Misericórdia de Franca) de $3 \mathrm{em} 3$ horas, com volume total de $150 \mathrm{~mL} / \mathrm{kg} / \mathrm{dia}$, que se apresentavam entre o $7^{\circ}$ e $10^{\circ}$ dias de vida, sem oxigênio suplementar e que pesavam até $2.500 \mathrm{~g}$.

Os RN com prematuridade extrema ou limítrofe, cardiopatia congênita, má-formação, doença pulmonar crônica, pósoperatório ou diagnóstico clínico de refluxo gastroesofágico foram excluídos. Fizeram também parte dos critérios de exclusão a utilização de via venosa para infusão, de antibioticoterapia e suporte ventilatório invasivo ou não-invasivo.

Comparações sequenciais foram executadas na mesma criança, seguindo os critérios de ensaios do tipo "crossover" ${ }^{(15)}$, no qual cada RN foi observado de forma alternada nas seguintes posições:

- decúbito lateral direito ( $L D)$;

- decúbito lateral esquerdo ( $L E)$;

- pronação $(P)$;

- supinação $(S)$.

As crianças foram posicionadas em $30^{\circ}$ de elevação e o posicionamento foi preconizado em 30 minutos antecedendo o início da gavagem. As sequências dos posicionamentos foram rotuladas em $1,2,3$ e 4 :

- sequência 1: LD, S, P e LE;

- sequência 2: $\mathrm{P}, \mathrm{LD}, \mathrm{LE}$ e $\mathrm{S}$;

- sequência 3: LE, P, S e LD;

- sequência 4: $\mathrm{S}, \mathrm{LE}, \mathrm{LD}$ e $\mathrm{P}$.

Através de sorteio, conhecia-se a sequência na qual o RN seria alocado. Foi estudada apenas uma criança por dia e apenas um posicionamento por gavagem, sendo o RN observado durante quatro gavagens seguidas. A coleta de dados foi realizada por um único examinador previamente treinado.

Informações clínicas foram obtidas através de consulta ao prontuário. A ficha foi composta pela identificação do RN e pelas variáveis: idade gestacional, data de nascimento, dias de vida, sexo, peso, índice de Apgar no $1^{\circ}$ e $5^{\circ}$ minuto de vida, uso de suporte pressórico invasivo, não-invasivo e/ou oxigênio suplementar, idade materna e tipo de parto.

Uma ficha com sinais clínicos foi utilizada durante as quatro gavagens intermitentes, onde foram anotadas as observações das seguintes variáveis dependentes: freqüência respiratória (FR), avaliada por ausculta e quantificada numericamente durante um minuto; frequência cardíaca (FC) e saturação de oxigênio $\left(\mathrm{SaO}_{2}\right)$, avaliadas através de sensor neonatal conectado a um oxímetro de pulso Oxypleth ("super bright"), Dixtal ${ }^{\circledR}$, DX 2405. A tiragem intercostal foi avaliada por inspeção e categorizada como: ausente, presente nos três últimos espaços intercostais ou presente em mais de três espaços intercostais; o batimento de asa nasal, avaliado por inspeção e categorizado como: ausente, discreto ou acentuado; a gemência, avaliada por ausculta e categorizada como: ausente, audível por estetoscópio ou audível sem estetoscópio e as intercorrências como: soluços, inquietação ou choro, foram anotadas ao lado de cada minuto, conforme ocorriam. Desde 5 minutos antes do início da alimentação até 5 minutos após o término, os dados foram coletados no intervalo de 2 em 2 minutos.

Foi utilizada a bomba infusora de seringa Samtronic ${ }^{\circledR}$ bomba de infusão 670, a extensão siliconada da marca Hartmann para a conexão entre a seringa de $20 \mathrm{~mL}$ e a sonda orogástrica número 6 - tubo de PVC atóxico - siliconado da Embramed. A bomba de infusão foi programada para o volume total da fórmula láctea ou leite materno ordenhado, sendo a velocidade de infusão $1 \mathrm{~mL}$ por minuto e o tempo variado ${ }^{(32)}$.

Comparações das médias das variáveis $\mathrm{FR}, \mathrm{FC}$ e $\mathrm{SaO}_{2}$ foram feitas por modelos de regressão linear de efeitos mistos $^{(13)}$, o que permitiu estimar as médias destas variáveis contínuas em cada posicionamento e em cada instante. As variáveis tiragem intercostal, batimento de asa nasal e gemência são de natureza qualitativa; assim, um modelo de regressão logística multinomial de efeitos mistos $^{(9)}$ foi utilizado para comparar as frequências relativas destas classificações entre as quatro posições do corpo. Estes modelos contêm um efeito aleatório, informando que cada $\mathrm{RN}$ participa de todos os posicionamentos e que suas medidas são tomadas longitudinalmente antes, durante e após a gavagem.

A análise dos dados foi conduzida no programa de computador SAS, sendo que em todos os testes de hipóteses construídos por estes modelos foi considerado um nível de significância de 5\%.

\section{RESULTADOS}

A Tabela 1 descreve as características individuais dos $\mathrm{RN}$ incluídos no estudo, bem como as médias e desvios-padrão das variáveis contínuas. 
TABELA 1. Características gerais dos RN estudados

\begin{tabular}{|c|c|c|c|c|c|c|c|c|c|c|c|c|}
\hline Número do RN & IG & $\begin{array}{c}\text { Dias de } \\
\text { vida }\end{array}$ & Sexo & $\begin{array}{c}\text { Peso ao } \\
\text { nascer } \\
\text { (g) }\end{array}$ & $\begin{array}{c}\text { Peso início } \\
\text { do estudo } \\
(\mathrm{g})\end{array}$ & $\begin{array}{l}\text { Apgar } 1^{\circ} \\
\min .\end{array}$ & $\begin{array}{c}\text { Apgar } 5^{\circ} \\
\text { min. }\end{array}$ & $\begin{array}{c}\text { VMI } \\
\text { (dias) }\end{array}$ & $\begin{array}{l}\text { VNI } \\
\text { (dias) }\end{array}$ & Parto & $\begin{array}{c}\text { Idade } \\
\text { materna } \\
\text { (anos) }\end{array}$ & Seq. \\
\hline 1 & 32 & 9 & $\mathrm{~F}$ & 1.605 & 1.430 & 5 & 9 & 3 & 4 & cesárea & 23 & 1 \\
\hline 2 & 34 & 7 & M & 1.880 & 1.810 & 7 & 9 & & & normal & 21 & 4 \\
\hline 3 & 31 & 9 & M & 1.580 & 1.460 & 8 & 9 & & & cesárea & 30 & 2 \\
\hline 4 & 31 & 10 & $\mathrm{~F}$ & 1.710 & 1.595 & 7 & 8 & & & cesárea & 17 & 3 \\
\hline 5 & 32 & 8 & M & 1.460 & 1.320 & 9 & 9 & & & normal & 23 & 4 \\
\hline 6 & 34 & 10 & M & 1.545 & 1.275 & 9 & 9 & 8 & & normal & 24 & 3 \\
\hline 7 & 33 & 8 & $\mathrm{~F}$ & 1.430 & 1.285 & 6 & 8 & & 1 & normal & 19 & 1 \\
\hline 8 & 31 & 10 & F & 1.715 & 1.455 & 4 & 7 & 2 & 3 & cesárea & 29 & 4 \\
\hline 9 & 34 & 7 & $\mathrm{~F}$ & 1.950 & 1.840 & 8 & 10 & & & normal & 35 & 2 \\
\hline 10 & 31 & 10 & M & 1.645 & 1.415 & 7 & 9 & & 9 & cesárea & 37 & 2 \\
\hline 11 & 31 & 10 & M & 1.680 & 1.440 & 6 & 8 & & 9 & normal & 27 & 2 \\
\hline 12 & 34 & 7 & M & 1.980 & 2.000 & 9 & 10 & & & cesárea & 17 & 1 \\
\hline 13 & 34 & 7 & $\mathrm{~F}$ & 2.500 & 2.450 & 6 & 8 & & & normal & 33 & 3 \\
\hline 14 & 32 & 8 & M & 1.360 & 1.270 & 7 & 9 & & & cesárea & 20 & 4 \\
\hline 15 & 33 & 7 & M & 1.620 & 1.585 & 9 & 9 & & & normal & 18 & 3 \\
\hline 16 & 31 & 10 & $\mathrm{~F}$ & 1.890 & 1.530 & 7 & 9 & & & cesárea & 25 & 1 \\
\hline Média & 32,4 & 8,6 & & 1.722 & 1.573 & 7,1 & 8,8 & 4,3 & 5,2 & & 24,8 & \\
\hline Desvio padrão & 1,31 & 1,31 & & 276,3 & 316,1 & 1,50 & 0,77 & 3,21 & 3,63 & & 6,41 & \\
\hline
\end{tabular}

$\mathrm{RN}=$ recém-nascido; IG = idade gestacional, em semanas

exo: classificado como $\mathrm{M}$ (masculino) e F (feminino);

作:

As Figuras 1, 2 e 3 ilustram as comparações entre os posicionamentos, onde as médias das $\mathrm{FR}, \mathrm{FC}$ e $\mathrm{SaO}_{2}$ respectivamente - foram estimadas pelos modelos de regressão linear de efeitos mistos, ajustadas por possíveis efeitos da idade gestacional, sexo do RN, peso ao nascer e forma de término de parto. Esses modelos testaram ainda possível efeito de sequências, ou seja, verificaram se a sequência de posicionamentos à qual o $\mathrm{RN}$ foi alocado poderia exercer algum efeito sobre os resultados obtidos. Não foram observados efeitos importantes resultantes desta alocação.

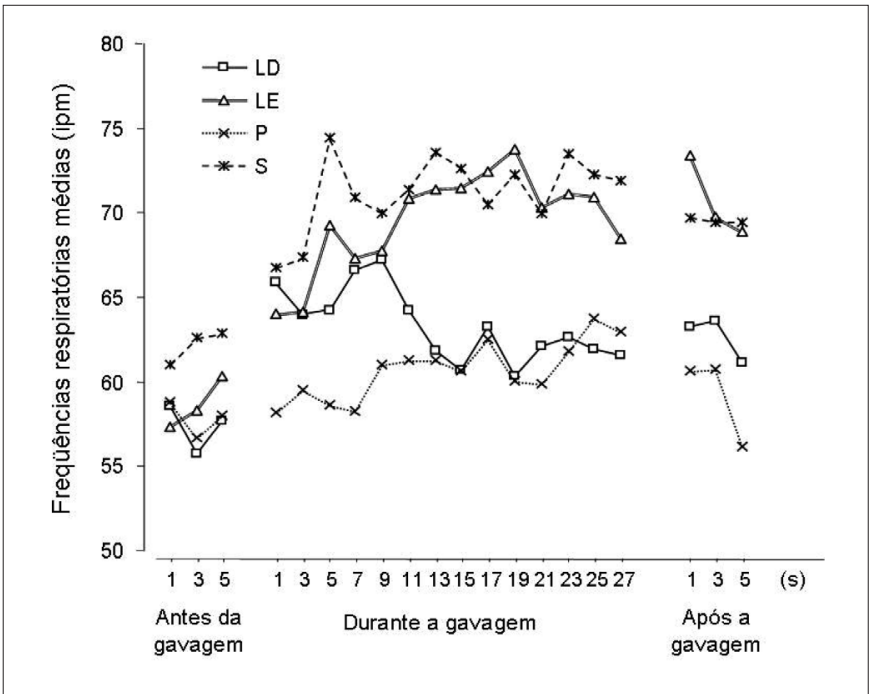

FIGURA 1. Comparações entre as frequências respiratórias médias (em incursões por minuto - ipm - ) ajustadas por idade gestacional, sexo do $\mathrm{RN}$, peso ao nascer e forma de término de parto

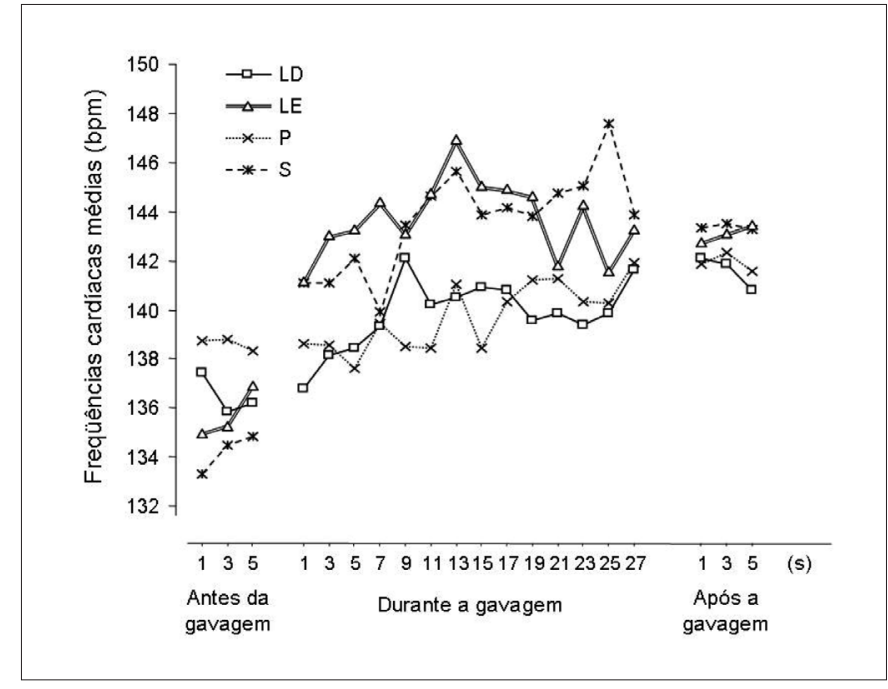

FIGURA 2. Comparações entre as frequências cardíacas médias (em bpm) dos tratamentos, ajustadas por idade gestacional, sexo do $\mathrm{RN}$, peso ao nascer e forma de término de parto

Observando as médias dos indicadores respiratórios vitais, nota-se (Figura 1) que as maiores diferenças de efeitos entre as FR médias dentro dos quatro posicionamentos ocorrem do $11^{\circ}$ ao $27^{\circ}$ minuto durante a gavagem, se comparadas às posições $\mathrm{P}$ e $\mathrm{S}, \mathrm{LE}$ e $\mathrm{P}, \mathrm{LD}$ e $\mathrm{S}$ e LD e LE. A FR média foi significantemente mais elevada antes do início da alimentação na posição em S, destacando-se entre todas as outras posições como o decúbito de maior repercussão negativa em repouso. De maneira geral (considerando todo o período), o posicionamento $\mathrm{S}$ trouxe maior FR média (69,9 incursões por minuto [ipm] 


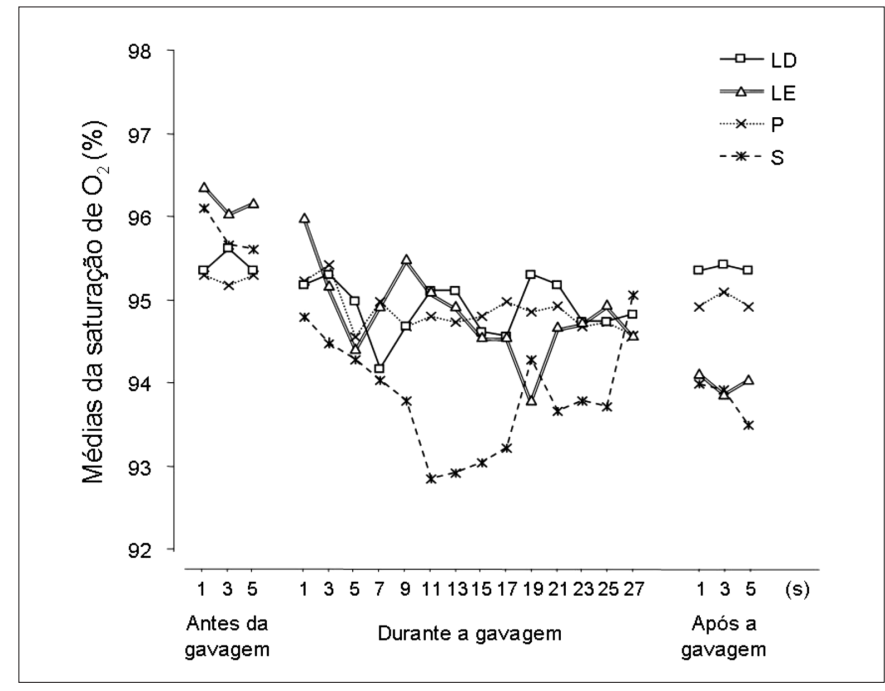

FIGURA 3. Comparações das saturações médias de oxigênio (\%) entre os tratamentos, ajustadas por idade gestacional, sexo do RN, peso ao nascer e forma de término de parto

com intervalo de confiança [IC] 95\% 65,3 a 74,2 (Tabela 2) seguido de LE, com média geral de 68,8 ipm (IC 95\% 64,3 a $73,2)$. Os posicionamentos $\mathrm{LD}$ e $\mathrm{P}$ apresentaram as menores médias gerais para a FR, respectivamente de 61,8 e 59,9 ipm.

TABELA 2. Comparações das médias gerais das frequências respiratórias dos RN (em ipm) entre os decúbitos

\begin{tabular}{llcccccc}
\hline \multirow{2}{*}{$\begin{array}{l}\text { Comparação } \\
\text { entre decúbitos }\end{array}$} & $\begin{array}{c}\text { Média } \\
\text { geral (ipm) }\end{array}$ & IC 95\% & \multicolumn{4}{c}{ Diferença (em ipm) entre as médias } \\
\cline { 6 - 8 } LD x LE & LD & 61,8 & $(57,366,2)$ & $-7,0$ & $(-9,2-4,7)$ & $<0,01$ \\
& LE & 68,8 & $(64,373,2)$ & & & & \\
LD x P & LD & 61,8 & $(57,366,2)$ & 1,9 & $(-0,4 \quad 4,2)$ & 0,11 \\
& P & 59,9 & $(55,464,3)$ & & & & \\
LD x S & LD & 61,8 & $(57,366,2)$ & $-8,0$ & $(-10,3-5,7)$ & $<0,01$ \\
& S & 69,8 & $(65,374,2)$ & & & & \\
LE x P & LE & 68,8 & $(64,373,2)$ & 8,9 & $(6,611,2)$ & $<0,01$ \\
& P & 59,9 & $(55,464,3)$ & & & & \\
LE x S & LE & 68,8 & $(64,373,2)$ & $-1,0$ & $(-3,2$ & $1,3)$ & 0,39 \\
& S & 69,8 & $(65,374,2)$ & & & & \\
P x S & P & 59,9 & $(55,464,3)$ & $-9,9$ & $(-12,1-7,6)$ & $<0,01$ \\
& S & 69,8 & $(65,374,2)$ & & & & \\
\hline
\end{tabular}

Ao se comparar entre si as médias gerais das FC (Tabela 3) nos quatro posicionamentos, não se encontrou nas combinações LD x P e LE x S, diferenças significativas $(P=$ $0,83)$ em ambas as comparações. Entretanto, comparando os posicionamentos LD x S, LD x LE, LE x P, P x S, houve diferenças significativas $(P<0,05$ em todas estas comparações (Tabela 3), embora a magnitude destas diferenças não seja muito importante do ponto de vista clínico (a maior diferença observada foi de 2,3 bpm) (Tabela 3). Na Figura 2 observa-se que LE e P mostram maiores diferenças no $5^{\circ}, 11^{\circ}, 13^{\circ}$ e $15^{\circ}$ minutos durante a gavagem. A resposta da FC média na análise dos posicionamentos destacou o
$\mathrm{LD}$ e $\mathrm{P}$ como sendo os decúbitos que responderam com menores valores, e o LE e $\mathrm{S}$ com os maiores valores (Figura 2 e Tabela 3 ).

TABELA 3. Comparações das médias gerais das frequências cardíacas dos RN (em bpm) entre os decúbitos

\begin{tabular}{|c|c|c|c|c|c|c|}
\hline \multirow{2}{*}{\multicolumn{2}{|c|}{$\begin{array}{l}\text { Comparação } \\
\text { entre decúbitos }\end{array}$}} & \multirow{3}{*}{$\begin{array}{c}\begin{array}{c}\text { Média } \\
\text { geral (bpm) }\end{array} \\
140,1\end{array}$} & \multirow{2}{*}{ IC $95 \%$} & \multicolumn{3}{|c|}{ Diferença (em bpm) entre as médias } \\
\hline & & & & \multirow{2}{*}{$\begin{array}{c}\text { Diferença } \\
-2,1\end{array}$} & IC $95 \%$ & \multirow{2}{*}{$\frac{P \text { valor }}{0,03}$} \\
\hline LD $x$ LE & LD & & $(132,9 \quad 147,3)$ & & $(-3,8-0,2)$ & \\
\hline & LE & 142,2 & $(135,0 \quad 149,4)$ & & & \\
\hline \multirow[t]{2}{*}{$L D \times P$} & LD & 140,1 & $(132,9 \quad 147,3)$ & $-0,2$ & $(-2,0 \quad 1,6)$ & 0,83 \\
\hline & $\mathrm{P}$ & 140,3 & $\left(\begin{array}{ll}133,1 & 147,5)\end{array}\right.$ & & & \\
\hline \multirow[t]{2}{*}{$\mathrm{LD} \times \mathrm{S}$} & LD & 140,1 & $(132,9 \quad 147,3)$ & $-2,3$ & $(-4,0-0,5)$ & 0,01 \\
\hline & S & 142,4 & $(135,2 \quad 149,6)$ & & & \\
\hline \multirow[t]{2}{*}{ LE $x$ P } & LE & 142,2 & $(135,0 \quad 149,4)$ & 1,9 & $(0,063,7)$ & 0,04 \\
\hline & $\mathrm{P}$ & 140,3 & $(133,1 \quad 147,5)$ & & & \\
\hline \multirow[t]{2}{*}{ LE $\times S$} & LE & 142,2 & $\left(\begin{array}{lll}135,0 & 149,4\end{array}\right)$ & $-0,2$ & $(-2,0 \quad 1,6)$ & 0,83 \\
\hline & S & 142,4 & $(135,2 \quad 149,6)$ & & & \\
\hline \multirow[t]{2}{*}{$\mathrm{P} \times \mathrm{S}$} & $\mathrm{P}$ & 140,3 & $(133,1 \quad 147,5)$ & $-2,1$ & $(-3,8-0,3)$ & 0,03 \\
\hline & S & 142,4 & $(135,2 \quad 149,6)$ & & & \\
\hline
\end{tabular}

IC = intervalo de confiança

Na comparação das médias gerais da saturação de oxigênio (Tabela 4), notam-se diferenças significativas apenas entre os decúbitos LD x S e LE x $\mathrm{S}(P<0,01$, em ambas comparações). No entanto, essas diferenças são pequenas e os respectivos IC (Tabela 4) sugerem que não são importantes do ponto de vista clínico (incluem somente valores de magnitude pequena). Quando considerados os instantes durante a alimentação, notam-se diferenças mais marcantes entre as posições LD e S (Figura 3) e entre LE e S (Figura 3) do $11^{\circ}$ ao $15^{\circ}$ minuto.

TABELA 4. Comparações das médias gerais da saturação de oxigênio dos RN (em percentagem) entre os decúbitos

\begin{tabular}{|c|c|c|c|c|c|c|c|c|}
\hline \multirow{2}{*}{\multicolumn{2}{|c|}{$\begin{array}{c}\text { Comparação } \\
\text { entre decúbitos }\end{array}$}} & \multirow{3}{*}{$\begin{array}{c}\begin{array}{c}\text { Média } \\
\text { geral }(\%)\end{array} \\
95,1\end{array}$} & \multirow{2}{*}{\multicolumn{2}{|c|}{ IC $95 \%$}} & \multicolumn{4}{|c|}{ Diferença (\%) entre as médias } \\
\hline & & & & & \multirow{2}{*}{$\frac{\text { Diferença }}{0,2}$} & \multicolumn{2}{|c|}{ IC $95 \%$} & \multirow{2}{*}{$\frac{P \text { valor }}{0,38}$} \\
\hline LD $x$ LE & LD & & $(94,2$ & $96,0)$ & & $(-0,2$ & $0,7)$ & \\
\hline & LE & 94,9 & $(94,0$ & $95,8)$ & & & & \\
\hline \multirow[t]{2}{*}{ LD $\times \mathrm{P}$} & LD & 95,1 & $(94,2$ & $96,0)$ & 0,4 & $(-0,02$ & (2),9) & 0,06 \\
\hline & $\mathrm{P}$ & 94,7 & $(93,8$ & $95,5)$ & & & & \\
\hline \multirow[t]{2}{*}{ LD x S } & LD & 95,1 & $(94,2$ & $96,0)$ & 0,8 & $(0,3$ & $1,3)$ & $<0,01$ \\
\hline & $\mathrm{S}$ & 94,3 & $(93,4$ & $95,1)$ & & & & \\
\hline \multirow[t]{2}{*}{ LE $\times$ P } & LE & 94,9 & $(94,0$ & $95,8)$ & 0,2 & $(-0,2$ & $0,7)$ & 0,32 \\
\hline & $\mathrm{P}$ & 94,7 & $(93,8$ & $95,5)$ & & & & \\
\hline \multirow[t]{2}{*}{ LE $\times S$} & LE & 94,9 & $(94,0$ & $95,8)$ & 0,6 & $(0,1$ & $1,1)$ & $<0,01$ \\
\hline & $\mathrm{S}$ & 94,3 & $(93,4$ & $95,1)$ & & & & \\
\hline \multirow[t]{2}{*}{$P \times S$} & $\mathrm{P}$ & 94,7 & $(93,8$ & $95,5)$ & 0,4 & $(-0,08$ & $0,9)$ & 0,11 \\
\hline & S & 94,3 & $(93,4$ & $95,1)$ & & & & \\
\hline
\end{tabular}

IC $=$ intervalo de confiança

Observa-se que o posicionamento em LD manteve níveis médios de $\mathrm{SaO}_{2}$ mais elevados durante e após a gavagem mesmo tendo uma $\mathrm{SaO}_{2}$ média antes do seu início, com níveis elevados (comparados aos outros posicionamentos).

A Figura 4 ilustra a frequência relativa de ausência de tiragens intercostais, presença nos três últimos espaços 
intercostais e em mais de três espaços, conforme o período da alimentação (antes, durante ou depois) e o posicionamento. Comparando-se essas frequências, evidencia-se diferença de efeitos $(P<0,01)$ em todas as combinações de posicionamentos, exceto quando se comparam os posicionamentos em LD $x$ $\mathrm{P}$ e LE x S. Contudo, o estudo mostrou evidências de que, conforme as frequências relativas de tiragens intercostais antes, durante e após a gavagem, os posicionamentos em $\mathrm{LD}$ e $\mathrm{P}$ foram os que mais apresentaram ausência de tiragem intercostal, sendo P o único que não apresentou tiragem intercostal em mais de três espaços intercostais no momento após a alimentação (Figura 4).

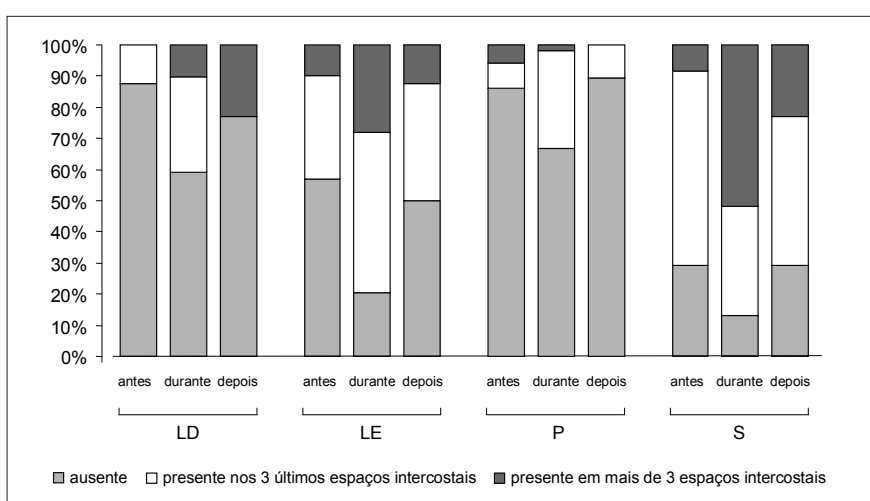

FIGURA 4. Tiragem intercostal, segundo os grupos, conforme o período da alimentação por gavagem (antes, durante ou depois)

Observa-se resposta semelhante com relação ao batimento de asa nasal (Figura 5). Nas frequências relativas de batimento de asa nasal antes, durante e após a gavagem, destacam-se os posicionamentos em LD e P, sendo estes os que mais apresentaram ausência deste sinal de desconforto respiratório. A Figura 5 mostra que a posição em $\mathrm{P}$ não apresentou nenhum sinal de batimento de asa nasal antes da alimentação. O mesmo ocorreu no posicionamento em LD após a alimentação.

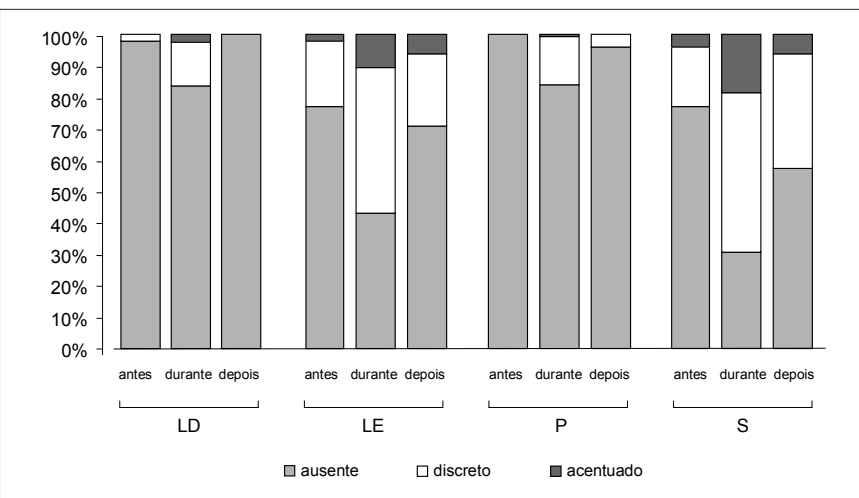

FIGURA 5. Batimento de asa nasal, segundo os grupos, conforme o período da alimentação por gavagem
Com respeito à ausência de gemência (Figura 6), audível com e sem estetoscópio, existiram dois grupos de posicionamentos em que não ocorreram diferenças significativas, sendo os posicionamentos LD x P e LE x S muito semelhantes entre si. Os posicionamentos em LE e $\mathrm{S}$ responderam com maior frequência de gemência audível com e sem estetoscópio antes, durante e após a gavagem (Figura 6).

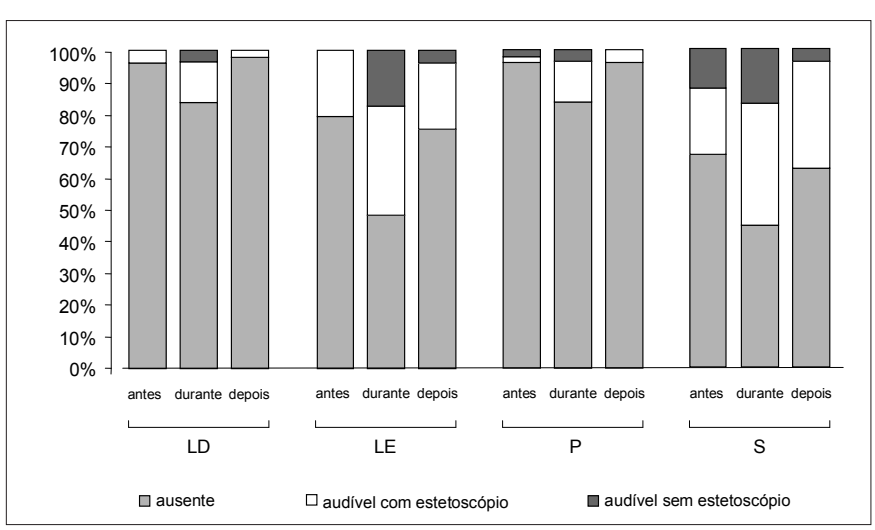

FIGURA 6. Gemência, segundo os grupos, conforme o período da alimentação por gavagem

\section{DISCUSSÃO}

Indicadores biológicos como FR, $\mathrm{FC} \mathrm{e} \mathrm{SaO}_{2}$ são utilizados para avaliar o grau de gravidade, determinar condutas e auxiliar a equipe de assistência à saúde a acompanhar a evolução da criança. Alguns indicadores podem sofrer alterações quando expostos à influência do posicionamento corporal. Assim, o decúbito postural participa diretamente no comportamento da ventilação e perfusão pulmonar. Destaca-se o efeito do posicionamento sobre a otimização do trabalho da musculatura respiratória, sendo que através do decúbito postural ocorre o ponto de apoio para a musculatura, favorecendo o seu desempenho e produzindo maior força de contração.

Desta forma, observa-se a desvantagem da biomecânica do sistema respiratório enfrentado pelo $\mathrm{RN}$, em que destaca o formato arredondado da parede torácica em vez de elíptico, como em adultos, resultando em arcos costais mais horizontalizados, alterando a relação comprimento-tensão dos músculos intercostais e diafragma, reduzindo-lhes a eficiência mecânica ${ }^{(11,17)}$. Associado a isso, esses arcos são pobremente mineralizados, o que culmina em uma complacência da parede torácica particularmente alta e oferece pouca estabilidade às diferentes forças de distorção, principalmente as resultantes de alterações de pressão intratorácica. A combinação desses fatores pode resultar em movimentos paradoxais da parede torácica durante a inspiração, reduzindo ainda mais a eficiência do movimento do diafragma, além de aumentar o trabalho desse músculo e torná-lo inapto em situações de aumento de demanda ventilatória ${ }^{(8,10,11,19)}$. 
Ao analisar a biomecânica do sistema respiratório observase a influência direta do sistema digestório, pois as vísceras abdominais como fígado, estômago e baço são separados do pulmão por meio da cúpula diafragmática. Entretanto, o aumento do volume gástrico após a alimentação em RN, provoca alterações no desempenho do músculo diafragma, o que refletirá em assincronismo toracoabdominal.

O posicionamento influencia na biomecânica do diafragma, pois sua contração depende de pontos fixos e móveis que o músculo sofre durante a posição corporal ${ }^{(25)}$. Estes pontos do diafragma são também influenciados pelo aumento do volume gástrico durante a dieta. Com o aumento do volume gástrico e da pressão intra-abdominal, o diafragma se retifica e o centro frênico passa para uma posição semi-fixa, provocando mudanças nos indicadores respiratórios.

Estudos anteriores demonstraram forte associação da alimentação intermitente por gavagem com aumento da frequência respiratória, associada ao aumento ou manutenção do volume minuto, visto que, com o aumento do volume gástrico, o pulmão sofre alterações na capacidade residual funcional ${ }^{(2,21)}$

Contudo, há posições (P e LD), como evidenciado na presente série, que não favorecem o aumento acentuado da FR, indicando maior conforto, menor instabilidade do sistema respiratório e menor gasto energético.

YU e ROLFE ${ }^{(28)}$ observaram que em $12 \mathrm{RN}$, o volume pulmonar total apresentou tendência a decréscimo e a FR a acréscimo após a alimentação. Por outro lado, LIOY e MANGINELLO ${ }^{(14)}$ relataram redução da FR nos RN em $\mathrm{P}$, comparados aos $\mathrm{RN}$ em $\mathrm{S}$, sendo este efeito também observado na presente casuística. A posição prona apresenta benefícios para a função respiratória, promovendo aumento da oxigenação, decréscimo do $\mathrm{CO}_{2}$ expirado, melhora da complacência, da função diafragmática e diminuição da assincronia toracoabdominal ${ }^{(20)}$.

Verificou-se que as posições em LE e $\mathrm{S}$ foram as que trouxeram maiores instabilidades ao sistema respiratório do grupo estudado, representando maior necessidade de ajuste para sustentação do volume/minuto. Entretanto, obteve-se também como achado neste estudo, o aumento da frequência respiratória no intervalo de 11 a 27 minutos no recebimento da alimentação, em todos os decúbitos. Não se encontraram na literatura estudos que corroboram este dado, mas podese perceber que é o momento de maior distensão gástrica.

$\mathrm{YU}^{(27)}$ sugeriu que os efeitos cardiorrespiratórios estavam relacionados ao deslocamento do volume da refeição na cavidade gástrica. $\mathrm{O}$ presente estudo ajustou a velocidade e o volume da refeição para cada $\mathrm{RN}$, contudo, os efeitos no sistema cardiorrespiratório apresentaram alterações frente aos posicionamentos adotados.

FIFER et al. ${ }^{(7)}$ observaram que a FC em $\mathrm{RN}$ foi mais elevada em $\mathrm{P}$, comparada à $\mathrm{S}$ durante a alimentação por gavagem, porém a variação da $\mathrm{FC}$ foi menor na posição prona. O mesmo foi observado neste artigo, em que a posição prona apresenta os valores mais elevados da FC média de repouso, embora se mantenha estáveis e mais baixos durante e após a alimentação.
Alguns estudos indicam que a queda da $\mathrm{SaO}_{2}$ e o aumento da $\mathrm{PCO}_{2}$ são gerados durante e/ou pós-gavagem ${ }^{(12,20)}$. Contudo, em alguns posicionamentos há repercussão da redução de $\mathrm{SaO}_{2}$, como constatado ao se observar o comportamento das posições em $\mathrm{P}$ e LD.

BOZYNSKI et al. ${ }^{(3)}$ observaram os efeitos no oxigênio arterial e nas medidas do dióxido de carbono do posicionamento em LD e LE em relação à posição $\mathrm{S}$, em $18 \mathrm{RN}$. O estudo concluiu que o posicionamento lateral não apresenta nenhum efeito na oxigenação e na ventilação, da mesma forma que no presente estudo.

A tiragem intercostal indica a presença de dificuldade na expansão pulmonar. Nesta casuística, as posições LD e P apresentaram maior ausência de tiragem intercostal, antes, durante a após a alimentação.

Assim, a posição de prona vem demonstrando vantagens no aumento da oxigenação arterial, no aumento do volume corrente e na melhora da função do diafragma, a qual reduz a incoordenação toracoabdominal. Evidências também vêm sendo relatadas com a associação da posição de prona e a redução mais acelerada dos parâmetros ventilatórios invasivos ${ }^{(1)}$.

Este resultado da $\mathrm{P}$ traz como justificativa a estabilidade da caixa torácica tanto pelo apoio do gradil costal como pelo aumento da pressão abdominal no apoio das vísceras na superfície do leito; desta forma, ocorre o melhor comprimento para a fibra muscular e potencialização do ângulo para a contração do músculo do diafragma, assim, desenvolvendo-se maior pressão transdiafragmática e melhora da ventilação pulmonar.

Nesta série, destacaram-se também no LD e na P, a menor ocorrência de batimento de asa nasal e o sinal de gemência, evidenciando menor trabalho respiratório e, consequentemente, do menor gasto energético. Estudos acrescentam que, durante a alimentação, o neonato deve ser mantido na posição de LD ou P, pois favorece e possibilita esvaziamento gástrico mais eficaz pós-gavagem ${ }^{(22,24,26)}$.

Já o posicionamento de S e LE apresentaram, neste estudo, maiores repercussões negativas no trabalho respiratório. Trabalho como de WOLFSON et al. ${ }^{(31)}$ descreve o achado com o posicionamento em $\mathrm{S}$, o que demonstra o assincronismo toracoabdominal e desvantagens da mecânica ventilatória como: distorção da caixa torácica, inspiração paradoxal, aumento do trabalho respiratório, fadiga e apneia em $\mathrm{RN}$.

Em S o tônus abdominal é insuficiente para gerar pressão intra-abdominal satisfatória e o diafragma trabalha com menor eficiência, porém, a contenção postural em flexão, utilizando rolos e coxins sob os ombros e membros inferiores, favorece uma postura fisiológica, aumenta o acoplamento toracoabdominal e, assim, a zona de aposição do diafragma e as desvantagens desta postura são amenizadas ${ }^{(6,29)}$. No presente estudo, os rolos e coxins foram utilizados para manter os RN prematuros na posição de $\mathrm{S}$, no entanto, os resultados mostraram evidências que, mesmo utilizando esses recursos, a posição $\mathrm{S}$ encontrou sinais de desconforto respiratório maior, obtendo também este achado no LE.

Por fim, considera-se a importância que o posicionamento exerce nos efeitos cardiorrespiratórios em $\mathrm{RN}$ que recebem 
a refeição por gavagem. Em conclusão, os LD e P exerceram menores desvantagens ao sistema cardiorrespiratório, provocando menor desconforto respiratório; já os LE e S foram os posicionamentos que demonstraram os maiores efeitos negativos no sistema cardiorrespiratório frente ao aumento do volume gástrico.

Pádua G, Martinez EZ, Brunherotti MAA. Cardiorespiratory effects of the body position in premature newborns submitted to the increase of the gastric volume. Arq Gastroenterol. 2009;46(4):321-7.

ABSTRACT - Context - The increase of the gastric volume observed in the gavage feeding is likely to cause consequences to the premature newborn, modifying the respiratory indicators. Objective - To investigate the alterations in the cardiorespiratory system of premature newborns submitted to an increase of the gastric volume by gavage feeding, according to four different body positioning methods. Methods - The study is a randomized crossover trial using a sample of 16 newborns with gestational age from 31 to 34 weeks and birth weight less or equal to $2.500 \mathrm{~g}$. The newborns were included in the study if they had from 7 to 10 days of life, feeding by orogastric tube, total volume of $150 \mathrm{~mL} / \mathrm{kg} /$ day and absence of supplemental oxygen-therapy. A different positioning method was used at each gavage (all raised to $30^{\circ}$ ), or say, right lateral, left lateral, prone and supine positions. The following response variables were considered: respiratory and cardiac frequencies, saturation of oxygen, drawing of intercostals, beating of nasal wing and grunting. These measures were collected in intervals of 2 minutes during 5 minutes after the gavage feeding, during whole period of the gavage feeding, and during 5 minutes before the gavage feeding. Results - The mean gestational age was 32 weeks (standard deviation 1.3) and the mean weight of the newborns was $1.722 \mathrm{~g}$ (standard deviation 276.3). The newborns presented higher values of the mean respiratory frequency in supine and left lateral body positions. In the right lateral and prone positions, the newborns presented lower mean cardiac frequency. The mean oxygen saturation had the lowest values in the left lateral and supine positions. The right lateral and prone positions presented low frequencies of intercostals drawing, beating of nasal wing and grunting. Conclusion - Our results indicate that right lateral and prone positions have influence on the cardiorespiratory effect, where left lateral and supine are the positions who presented higher negative effect in the newborns submitted to the increase of the gastric volume.

HEADINGS - Infant, premature. Posture. Cardiovascular system. Respiration. Enteral nutrition.

\section{REFERÊNCIAS}

1. Antunes LCO, Rugolo LMSS, Crocci AJ. Efeito da posição do prematuro no desmame da ventilação mecânica. J Pediatr (Rio J). 2003;79:239-44.

2. Barrie H. Effect of feeding on gastric and esophageal pressures in the newborn. Lancet. 1968;2:1158-60.

3. Bozynski MEA, Naglie RA, Nicks JJ, Burpee B, Johnson RV. Lateral positioning of the stable ventilated very-low-birth-weight infant. Effect on transcutaneous oxygen and carbon dioxide. Am J Dis Child. 1988;142:200-2.

4. Brar G, Geiss D, Brion LP, Rios A. Respiratory mechanics in very low birth weight infants during continuous versus intermittent gavage feeds. Pediatr Pulmonol. 2001;32:442-6.

5. Capurro H, Konichezky S, Fonseca D, Caldeyrobarcia R. Simplified method for diagnosis of gestational age in newborn-infant. J Pediatr. 1978;93:120-2.

6. Dominguez SS, Komiyama S. Cuidados fisioterápicos ao recém-nascido em ventilação mecânica. In: Kopelman B, Miyoshi MH. Guinsburg R, editores. Distúrbios respiratórios no período neonatal. São Paulo: Atheneu; 1998. p.527-41

7. Fifer WP, Myers MM, Sahni R, Ohira-kist K, Kashyap S, Stark RI, Schulze KF. Interactions between sleeping position and feeding on cardiorespiratory activity in preterm infants. Dev Psychobiol. 2005;47:288-96.

8. Flemming PJ, Muller NL, Bryan MH, Bryan AC. The effects of abdominal loading on rib cage distortion in premature infants. Pediatrics. 1979;64:425-8.

9. Hedeker D. A mixed-effects multinomial logistic regression model. Statist Med 2003;22:1433-46.

10. Heldt GP, McIlroy MB. Dynamics of chest wall in preterm infants. J Appl Physiol. 1987;62:170-4.

11. Heldt GP. Development of stability of the respiratory system in preterm infants. J Appl Physiol. 1988;65:441-4.

12. Heldt GP. The effect of gavage feeding on the mechanics of lung, chest wall, and diaphragm of preterm infants. Pediatr Res. 1988;24:55-8.

13. Laird NM, Ware JH. Random-effects models for longitudinal data. Biometrics. 1982;38:963-74.

14. Lioy J, Manginello FP. A comparison of prone and supine positioning in the immediate postextubation period of neonates. J Pediatr. 1988;112:982-4.

15. Pocock SJ. Clinical trials: a practical approach. Chichester: John Wiley, 1983.

16. Miyoshi MH, Guinsburg R, Kopelman B. Síndrome do desconforto respiratório do recém-nascido. In: Kolpeman B, Miyoshi MH, Guinsburg R, editores. Distúrbios respiratórios no período neonatal. São Paulo: Atheneu; 1998. p.63-74.

17. Mortola JP, Saetta M, Fox G, Smith B, Weeks S. Mechanical aspects of chest wall distortion. J Appl Physiol. 1985;59:295-304.
18. Muller KE, Stewart PW. Linear model theory: univariate, multivariate and mixed models. Hoboken: John Wiley; 2006.

19. Papastamelos C, Panitch HB, England SE, Allen JL. Developmental changes in chest wall compliance in infancy and early childhood. J Appl Physiol. 1995; 78:179-84.

20. Patel BD, Dinwiddie R, Kumar SP, Fox WW. The effects of feeding on arterial blood gases and lung mechanics in newborn infants recovering from respiratory disease. J Pediatr. 1977;90:435-8.

21. Pitcher-Wilmott R, Shutack JG, Fox WW. Decreased lung volume after nasogastric feeding of neonates recovering from respiratory disease. J Pediatr. 1979;95:119-21.

22. Price PT, Kalhan SC. Nutrição para o neonato de alto risco. In: Klaus MH, Fanaroff AA, editores. Alto risco em neonatologia. $4^{\mathrm{a}}$ edição. Rio de Janeiro: Guanabara Koogan; 1995. p.98-129.

23. Segre CAM. Recém-nascido pré-termo. In: Segre CAM, editor. Perinatologia: fundamentos e prática. São Paulo: Sarvier; 2002. p.232-51.

24. Silva MHA, Scochi CGS, Kokuday MLP, Sprioli RMD, Silva-Netto KA Alimentação do bebê prematuro e de muito baixo peso ao nascer: subsídios para a assistência de enfermagem em berçário. Pediatr Mod. 2000;36:282; 286; 290 passim - 283-288-291.

25. Souchard PE. Respiração. In: Souchard PE. O diafragma. $4^{a}$ edição. São Paulo: Summus; 1990. p.11-31.

26. Yu VY. Effect of body position on gastric emptying in the neonate. Arch Dis Child. 1975:50:500-4.

27. Yu VY. Cardiorespiratory response to feeding in newborn infants. Arch Dis Child. 1976;51:305-9.

28. Yu VY, Rolfe P. Effect of feeding on ventilation and respiratory mechanics in newborn infants. Arch Dis Child. 1976;51:310-3.

29. Yunes AP, Talerman C. Fisioterapia. In: Segre CAM, editor. Perinatologia: fundamentos e prática. São Paulo: Savier; 2002. p.815-9.

30. West JB. Mecânica da respiração: Como o pulmão é sustentado e movimentado. In: West JB. Fisiologia respiratória. $6^{\text {a }}$ edição. Barueri, SP: Manole; 2002. p.89116

31. Wolfson MR, Greenspan JS, Deoras KS, Allen JL, Shaffer TH. Effect of position on the mechanical interaction between the rib cage and abdomen in preterm infants. J Appl Physiol. 1992;72:1032-8.

32. Wong DL. Enfermagem pediátrica - elementos essenciais à intervenção efetiva. $5^{\text {a }}$ edição. Rio de Janeiro: Guanabara Koogan; 1999. p.658-9.

Recebido em 31/3/2008 Aprovado em10/3/2009. 